\title{
IDEALS AND CENTRALIZING MAPPINGS IN PRIME RINGS
}

\author{
JOSEPH H. MAYNE
}

\begin{abstract}
Let $R$ be a prime ring and $U$ be a nonzero ideal of $R$. If $T$ is a nontrivial automorphism or derivation of $R$ such that $u u^{T}-u^{T} u$ is in the center of $R$ and $u^{T}$ is in $U$ for every $u$ in $U$, then $R$ is commutative. If $R$ does not have characteristic equal to two, then $U$ need only be a nonzero Jordan ideal.
\end{abstract}

If $R$ is a ring, a mapping $T$ of $R$ to itself is called centralizing on a subset $S$ of $R$ if $s s^{T}-s^{T} s$ is in the center of $R$ for every $s$ in $S$. There has been considerable interest in centralizing automorphisms and derivations defined on rings. Miers [4] has studied these mappings defined on $C^{*}$-algebras. In [5] Posner proved that if a prime ring has a nontrivial centralizing derivation, then the ring must be commutative. The same result was obtained for centralizing automorphisms in [3]. In this paper it is shown that the automorphism or derivation need only be centralizing and invariant on a nonzero ideal in the prime ring in order to ensure that the ring is commutative. Also, if $R$ is of characteristic not two, then the mapping need only be centralizing and invariant on a nonzero Jordan ideal. For derivations this gives a short proof of a result related to that of Awtar [1, Theorem 3]. Awtar proved that if $R$ is a prime ring of characteristic not two with a nontrivial derivation and a nonzero Jordan ideal $U$ such that the derivation is centralizing on $U$, then $U$ is contained in the center of $R$.

Jeffrey Bergen deserves many thanks for his suggestions concerning the results and proofs in this paper.

From now on assume that $R$ is a prime ring and let $Z$ be the center of $R$. Let $[x, y]=x y-y x$ and note the important identity $[x, y z]=y[x, z]+[x, y] z$. The following lemmas will be used in the proofs of the main results.

LEMMA 1. If $b[a, r]=0$ for all $r$ in $R$, then $b=0$ or $a$ is in $Z$.

PROOF. Assume that $b[a, r]=0$ for all $r$ in $R$. Replace $r$ by $x y$ to obtain $b[a, x y]=b x[a, y]+b[a, x] y=b x[a, y]=0$ for all $x$ and $y$ in $R$. Since $R$ is prime, $b=0$ or $[a, y]=0$ for all $x$ in $R$.

LEMMA 2. If $D$ is a derivation of $R$ such that $u^{D}=0$ for all $u$ in a nonzero right ideal $U$ of $R$, then $r^{D}=0$ for all $r$ in $R$.

Proof. Let $u$ be a nonzero element in $U$ and $x$ be an element in $R$. Then $u x$ is in $U$ and $0=(u x)^{D}=u^{D} x+u\left(x^{D}\right)=u\left(x^{D}\right)$. Now replace $x$ by $s r$ to obtain $0=u(s r)^{D}=\left[u\left(s^{D}\right)\right] r+u s\left(r^{D}\right)=u s\left(r^{D}\right)$ for all $r$ and $s$ in $R$. Since $R$ is prime and $u$ is nonzero, $r^{D}=0$ for all $r$ in $R$.

Received by the editors October 21, 1981 and, in revised form, January 12, 1982.

1980 Mathematics Subject Classification. Primary 16A70; Secondary 16A12, 16A68, 16A72.

Key words and phrases. Ideal, centralizing automorphism, centralizing derivation, prime ring, commutative. 
LEMMA 3. If $T$ is a homomorphism of $R$ such that $u^{T}=u$ for all $u$ in a nonzero right ideal $U$ of $R$, then $r^{T}=r$ for every $r$ in $R$.

Proof. Let $u$ be a nonzero element in $U$ and $r, s$ be in $R$. Since $U$ is a right ideal, $u s$ and $u s r$ are in $U$. Then $(u s r)^{T}=u s r=(u s)^{T} r^{T}=u s r^{T}$. Hence $u s\left(r-r^{T}\right)=0$ for all $s$ and $r$ in $R$. Thus $r=r^{T}$ for all $r$ in $R$.

LEMMA 4. If $R$ contains a nonzero commutative right ideal $U$, then $R$ must be commutative.

Proof. Let $u$ be in $U$ and assume that $u^{2}$ is not zero. Such an element exists for if not, then by a variation of Levitzki's theorem [2, Lemma 1.1], $R$ has a nonzero nilpotent ideal and this is impossible in a prime ring. $U$ is a right ideal and so ur and $u s$ are in $U$ for every $r$ and $s$ in $R$. Since $U$ is commutative, $u^{2} s r=u(u s) r=$ $u s(u r)=u r(u s)=u(u r) s=u^{2} r s$. Hence $u^{2}[r, s]=0$ for all $r$ and $s$ in $R$. By Lemma 1 , every $r$ in $R$ is in $Z$. Therefore $R$ is commutative.

THEOREM. Let $R$ be a prime ring and $U$ be a nonzero ideal of $R$. If $R$ has a nontrivial automorphism or derivation $T$ such that $u u^{T}-u^{T} u$ is in the center of $R$ and $u^{T}$ is in $U$ for every $u$ in $U$, then $R$ is commutative.

Proof. By Lemma 2 or Lemma 3, $T$ is nontrivial on $U$. Since $U$ is a nonzero ideal in a prime ring, $U$ is itself a prime ring. $U$ is then commutative by the author's result in [3] for automorphisms or by Posner's result [5] for derivations. By Lemma $4, R$ is commutative.

COROLLARY. If $U$ is a nonzero Jordan ideal in a prime ring $R$ of characteristic not two and $T$ is a nontrivial automorphism or derivation of $R$ which is centralizing and invariant on $U$, then $R$ is commutative.

PrOOF. Every nonzero Jordan ideal in a prime ring of characteristic not two contains a nonzero ideal [2, Theorem 1.1]. Apply the theorem to this ideal.

\section{REFERENCES}

1. R. Awtar, Lie and Jordan structures in prime rings with derivations, Proc. Amer. Math. Soc. 41 (1973), 67-74.

2. I. Herstein, Topics in ring theory, Univ. of Chicago Press, Chicago, Illinois, 1969.

3. J. Mayne, Centralizing automorphisms of prime rings, Canad. Math. Bull. 19 (1976), 113-115.

4. C. R. Miers, Centralizing mappings of operator algebras, J. Algebra 59 (1979), $56-64$.

5. E. Posner, Derivations in prime rings, Proc. Amer. Math. Soc. 8 (1957), 1093-1100.

Department of Mathematical Sciences, loyola University, Chicago, ILLINOIS 60626 